\title{
Amniotic Fluid Embolism: A Rare Cause of Maternal Collapse: A Case Report
}

\author{
Sarita Sitaula ${ }^{1}$, Dipti Das ${ }^{1}$, Subhas Sitaula ${ }^{2}$, and Manisha Chhetry ${ }^{1}$ \\ ${ }^{1}$ BP Koirala Institute of Health Sciences \\ ${ }^{2}$ Singing River Health System
}

September 11, 2020

\begin{abstract}
We present a case of postpartum women who collapsed immediately after delivery with probable diagnosis of amniotic fluid embolism. This is a rare but fatal condition and was managed with supportive measures. Suspicion of amniotic fluid embolism should be considered in any postpartum collapse if no obvious cause is known.
\end{abstract}

\section{Amniotic Fluid Embolism: A Rare Cause of Maternal Collapse: A Case Report}

Sarita Sitaula ${ }^{1}$, Dipti Das ${ }^{1}$, Subhas Sitaula ${ }^{2}$, Manisha Chhetry ${ }^{1}$

1 B.P. Koirala institute of Health Sciences Dharan, Nepal

2 Critical Care Unit, Singing River Health System, Mississippi USA

Corresponding author

Dr Sarita Sitaula

Assistant Professor, BPKIHS, Dharan, Nepal

Email: dr.sarita.sitaula@gmail.com, sarita.sitaula@bpkihs.edu

ORCID iD : https://orcid.org/0000-0001-5312-4590

Contact number: +977-9842052514

Abstract

We present a case of postpartum women who collapsed immediately after delivery with probable diagnosis of amniotic fluid embolism. This is a rare but fatal condition and was managed with supportive measures. Suspicion of amniotic fluid embolism should be considered in any postpartum collapse if no obvious cause is known.

Keywords:

Amniotic fluid embolism, Maternal mortality/ collapse, Postpartum collapse, Coagulopathy

Key clinical message

High index of suspicion of amniotic fluid embolism should be considered in any intrapartum or postpartum collapse where obvious cause of collapse is not identified.

Introduction: 
Amniotic fluid embolism (AFE) is a rare complication of pregnancy, associated with significant morbidity and mortality, occurring in 2-8 per 100,000 pregnancies[1]. They usually present with sudden and unexplained cardiorespiratory collapse and disseminated intravascular coagulopathy [2]. This is one of the cause for sudden death in obstetrics with high mortality rate, ranging from 20 to 60\% [3]. Early recognition and prompt resuscitation are the key components for the management of AFE.

Case presentation

A 30 year old, unbooked, primigravida at 39 weeks 6 days of period of gestation (POG) presented to obstetric emergency of BPKIHS, Nepal, referred from private hospital with diagnosis of intrauterine fetal death (IUFD) with obstetric cholestasis.

She had stopped perceiving fetal movements for 2 days. She gave history of itching of the whole body, which had started at 35 weeks of gestation. Her liver function showed increased in liver enzymes( SGPT/SGOT$224 / 204 \mathrm{IU} / \mathrm{L}$ respectively) and diagnosis of obstetric cholestasis was made clinically as bile acid concentration measurement was not available. She was started with tablet Ursodeoxycholic acid $300 \mathrm{mg}$ BD for 1 week and then she stopped the medication on her own.

Her $1^{\text {st }}$ and $2^{\text {nd }}$ trimester was uneventful and no other significant past and family history was present.

She is a non-smoker and non-alcoholic.

On examination at admission:

She was well oriented to time, place and person. Her vitals were within normal limits, with systolic blood pressure of $110 \mathrm{mmHg}$ and diastolic $70 \mathrm{mmHg}$. Abdominal examination showed term size fetus in cephalic presentation without cardiac activity and patient was not in labor (BISHOP score-3).

Her investigation reports at admission were all normal except increased liver enzymes value.

She was induced with $50 \mathrm{mcg}$ of misoprostol, which was kept per-vaginum 4 hourly on $2^{\text {nd }}$ day of admission. She had progressed after $3^{\text {rd }}$ dose of misoprostol and delivered a macerated female weighing $2.9 \mathrm{~kg}$ after 12 hours of induction. Labor and delivery were uneventful.

Around half an hour of the delivery, she had started having symptoms of hypoxia like: irritability, sweating, anxiety. On examination, she was tachycardic and tachypneic with pulse rate 170 beats per minute and respiratory rate 24 breaths per minute respectively. Oxygen saturation was up to $92 \%$ on room air. There was drop in the blood pressure and became not recordable within a few minutes. Patient was conscious throughout with GCS of 15/15. Abdominal and local examination were normal and there was no evidence of postpartum hemorrhage or on going blood loss. Resuscitation was started immediately with intravenous fluid and oxygen supplementation. Repeat blood investigations were sent, blood products were arranged and patient was shifted to ICU. After $1500 \mathrm{ml}$ of fluid resuscitation blood pressure was recorded up to 80/40 $\mathrm{mmHg}$ but there was no urine output. Arterial blood gas analysis showed lactic acidosis with metabolic alkalosis. Patient was started with noradrenaline as she was not maintaining blood pressure which was continued for 36 hours and stopped gradually. Patient had started bleeding and soakage from episiotomy site hence, adrenaline packing was also done. Investigation reports were collected which was suggestive of DIC. She was transfused with IV unit of fresh frozen plasma, IV units of fresh blood and I unit of whole blood over 48 hours. Her renal function was deranged and creatinine values worsened in the first three days. However, as she had started passing urine after 8 hours of resuscitative measure, she didn't require hemodialysis. She was also started with broad spectrum antibiotics and low molecular heparin which was continued for 10 days.

Patient developed tachypnea on $4^{\text {th }}$ postpartum day. Chest x-ray was done and was suggestive of pleural effusion. Diagnostic pleural fluid tapping was performed, which was suggestive of transudative effusion. Echocardiography, D- dimer and APTT was normal whereas electrocardiogram showed sinus tachycardia.

Multidisciplinary management of the patient was done in the maternal ICU for 8 days. She was then 
transferred to ward on $8^{\text {th }}$ day as she was improving clinically and was discharged from hospital on $14^{\text {th }}$ day. Patient was doing fine at $3^{\text {rd }}$ week follow up after discharge.

\section{Discussion}

AFE is a rare complication but has high fatality rate, characterized by sudden cardiovascular collapse, dyspnea or respiratory collapse and disseminated intravascular coagulopathy[4]. This may occur in healthy women during pregnancy, labor or following delivery. It can develop even after elective abortion, amniocentesis, cesarean delivery or trauma. This condition is initiated by entry of amniotic fluid to the blood stream of the mother which leads a serious reaction causing cardiopulmonary arrest and massive coagulopathy[3][5].

Because AFE is a diagnosis of exclusion, a precise case definition and criteria is difficult to establish and also other causes of maternal collapse should be ruled out. The causes of maternal collapse could be due to hemorrhagic shock, pulmonary embolism, anaphylaxis, septic shock, aortic dissection [6]. Diagnosis is based upon the signs and symptoms observed during the birth or procedure.

The associated risk factors for AFE are age more than 35 years, multiparity, cesarean section, instrumental delivery, antepartum hemorrhage, eclampsia, labor induction, fetal distress, fetal death, male baby [7][8] [9].

Few authors have proposed two clinical forms of AFE typical and atypical. Typical or classic form has three phases: phase 1 - respiratory and circulatory disorders, phase 2- coagulation disturbances and phase 3 -acute renal failure and acute respiratory distress syndrome(ARDS) leading to cardiopulmonary collapse[10]. In Atypical form, cardiopulmonary collapse doesn't occur but first symptom is life threatening hemorrhage due to DIC[11].

The most significant diagnosis of AFE is made by findings at autopsy, which are limited to the lungs and clinical diagnostic criteria, and assisted by serum markers[12]. Serum markers like C3, C4 and C1 esterase inhibitors are reduced.

The symptoms are usually sudden in onset. Acute dyspnea, agitation, sudden chills, sweating coughing and anxiety are common premonitory symptoms. Labored breathing and tachypnea may occur. Diagnosis can be made by following criteria[2][13],

1. Acute hypotension or cardiac arrest

2. Acute hypoxia

3. Coagulopathy or severe hemorrhage in the absence of other explanations

4. All of these occurring during labor, cesarean delivery, dilation and evacuation or within 30 minute postpartum with no other explanation of findings.

In our case, this women had risk factor of fetal death and labor induction. She didn't have fever, obstetric hemorrhage, eclampsia or any other identifiable cause of postpartum collapse and also, she fitted to the above mentioned criteria of AFE, hence we had made diagnosis of AFE.

The initial phase of AFE consists mainly of right ventricular failure. If available, transthoracic and or transesophageal echocardiography may provide valuable information. Immediately after presentation, the echocardiography will reveal a severely dilated hypokinetic right ventricle (acute Cor pulmonale) with deviation of the interventricular septum in to the left ventricle. In our case transthoracic echocardiography was done on $3^{\text {rd }}$ day which showed mild mitral regurgitation, mild pulmonary artery hypertension without any vegetation and clots. As patient was already stabilized, finding may not be suggestive of AFE or pulmonary embolism. Also, early and aggressive resuscitation with blood product to correct coagulopathy results in improved outcomes[14].

As computed tomography angiography or ventilation perfusion scan is not available in our center, we were not able to perform this test, which could have been performed to rule out pulmonary embolism. As this patient had coagulopathy, acute hypoxia and maternal collapse which is in favor of AFE than pulmonary embolism. 
Survival after AFE has improved significantly due to early recognition and management, morbidity remains high with severe sequelae. Neurological impairment is most common complication followed by renal failure, cardiac failure with left ventricular impairment, arrythmia have been reported [8].

Primary management is respiratory support and hemodynamic support with judicious use of fluids, vasopressors, inotropes and pulmonary vasodilators. Early diagnosis and aggressive management of patient with resuscitation improves the survival as well as long term morbidities.

\section{Contributors:}

SS1, DD were involved in diagnosing the patient. SS1, DD were involved in treatment and management of the patient. SS1 wrote majority of the manuscript and formulated the hypothesis. SS2 helped and guided during patient diagnosis and management.MC helped in formulating the manuscript. All co-authors provided critical feedback and helped shape the research, analysis and manuscript.

\section{Declaration of Competing Interest}

The authors declare that they have no conflict of interest regarding the publication of this case report.

\section{Funding}

No specific grant from any funding agency supported the publication of this case report.

\section{Ethical approval}

As this was a case report ethical approval from Institutional Review Committee was not sought. However, written informed consent was obtained from the patient.

\section{Patient consent}

Written consent for publication was obtained from the patient.

Acknowledgements : We thank the patient and her husband in co-operating with the history taking and the nursing staff in managing the patient during the hospital stay. We would also like to thank Dr. Pawan Mahat for providing valuable feedback in structuring the manuscript.

\section{References}

1. Lisonkova S, Kramer MS. 2019. Amniotic fluid embolism: A puzzling and dangerous obstetric problem. PLoS Med. 16(11):11-3.

2. Clark SL, Hankins GDV, Dudley DA, Dildy GA, Porter TF. 1995. Amniotic fluid embolism: Analysis of the national registry. Am J Obstet Gynecol:1158-69.

3. Clark SL.2014. Amniotic fluid embolism. Obstet Gynecol. (123):337-48.

4. Lee JH, Yang HJ, Kim JH, Lee SY, Gill HJ, Kim BK, et al.2010. Amniotic fluid embolism that took place during an emergent Cesarean section. Korean J Anesthesiol. 59(SUPPL.):158-62.

5. Gilmore DA, Wakim J, Secrest J, Rawson R.2003. Anaphylactoid syndrome of pregnancy: a review of the literature with latest management and outcome data. AANA J. 71(2):120-126.

6. Benson MD.2012. Current concepts of immunology and diagnosis in amniotic fluid embolism. Clin Dev Immunol: 2012.

7. Abenhaim HA, Azoulay L, Kramer MS, Leduc L.2008. Incidence and risk factors of amniotic fluid embolisms: a population-based study on 3 million births in the United States. Am J Obstet Gynecol. (199):49.e1-49.e8.

8. Fitzpatrick KE, Van Den Akker T, Bloemenkamp KWM, Deneux-Tharaux C, Kristufkova A, Li Z, et al. 2019. Risk factors, management, and outcomes of amniotic fluid embolism: A multicountry, population-based cohort and nested casecontrol study. PLoS Med. 16(11):1-24.

9. Toy H. Amniotic fluid embolism. 2009. Eur J Gen Med. (6):108-15. [Google Scholar]

10. Uszynski M. 2009. Amniotic fluid embolism: the complication of known pathomechanism but without pathogenetic therapy? Thromb Haemost.101(5):795-6.[PubMed] 
11. Uszyński M. 2011. Amniotic fluid embolism: literature review and an integrated concept of pathomechanism. Open J Obstet Gynecol. 01(04):178-83.

12. Kanayama N, Tamura N. Amniotic fluid embolism:2014. Pathophysiology and new strategies for management. Vol. 40, J Obstet and Gynaecol Res. (40) :1507-17.

13. O'Shea A, Eappen S (2007). Amniotic Fluid Embolism, International Anesthesiology Clinics. (45):1728.

14. Pacheco LD, Saade G, Hankins GDV, Clark SL.2016. Amniotic fluid embolism: diagnosis and management. Am J Obstet Gynecol. 215(2):B16-24. 